\title{
Effect of CCK-8 on intake of caffeine, ethanol, and water
}

\author{
PAUL J. KULKOSKY, W. ERIC HOLST, WENDY G. SMITH, and MAX A. DIETZE \\ University of Southern Colorado, Pueblo, Colorado
}

\begin{abstract}
Three groups of 10 water-deprived female rats received access to solutions of caffeine $(0.5 \mathrm{mg} / \mathrm{ml})$, ethanol (5\% w/v), or water, after i.p. injection of the neuropeptide and hormone cholecystokinin octapeptide (CCK-8; 0.5-4.0 $\mu \mathrm{g} / \mathrm{kg}$ ). Behavioral displays associated with intake of these fluids were quantified with an instantaneous time-sampling observational technique. CCK-8 dose-dependently reduced intake of ethanol solution, but not intake of caffeine solution or water. Feeding behavior reliably $(p<.05)$ decreased after caffeine consumption, and activity level increased. Chemically induced anorexias were specific to ingesta; CCK-8 did not affect caffeine or water intake, or caffeineinduced anorexia, but it did inhibit ethanol intake and associated feeding behavior.
\end{abstract}

Cholecystokinin octapeptide (CCK-8) is a gut hormone and neural peptide that is known to inhibit food intake (Gibbs, Young, \& Smith, 1973) and ethanol intake (Kulkosky, 1984) after parenteral injection. CCK-8 has been hypothesized to act as an endogenous signal of satiation in the short-term control of feeding (Gibbs \& Smith, 1991; Smith, 1984; Smith \& Gibbs, 1988) and alcohol consumption (Kulkosky, 1985). Caffeine is a commonly consumed methyl xanthine drug that also inhibits food and alcohol intakes after intraperitoneal (i.p.) injection at high doses (Dietze \& Kulkosky, 1991; Hederra, Aldunate, Segovia-Riquelme, \& Mardones, 1975; McKim, 1980).

One criterion of an endogenous satiety factor is motivational specificity of action; intakes of caloric sources and water are not equally suppressed by exogenous administration of the factor (Gibbs et al., 1973; Kulkosky, Sanchez, Foderaro, \& Chiu, 1989; Mueller \& Hsiao, 1977; Smith, 1984). In the following experiment, we tested the specificity of CCK-8's inhibitory effect on ingestion by comparing its action on consumption of caffeine solution, alcohol solution, and water. The behavioral displays accompanying these fluid intakes were also quantified with a reliable and valid instantaneous time-sampling observational technique (Antin, Gibbs, Holt, Young, \& Smith, 1975). These procedures allowed determinations of whether or not orally consumed caffeine inhibited feeding behavior, and whether or not CCK- 8 could affect the intake of this noncaloric psychoactive drug through a general "drug satiety" action.

This research was supported by Grant 2 S06 RR08197-09 from The Division of Research Resources, NIH, and NIAAA, and was first presented at the 31st Annual Meeting of the Psychonomic Society in New Orleans, 1990. The authors thank the Squibb Institute for Medical Research for providing sulfated octapeptide of cholecystokinin (SQ 19,844). Max A. Dietze is currently in the Department of Psychology, Fort Hays State University, Hays, KS 67601. Correspondence should be addressed to Paul J. Kulkosky, Department of Psychology, University of Southern Colorado, Pueblo, CO 81001-4901.

\section{METHOD}

\section{Subjects}

The subjects were 30 experimentally naive, female Charles River Wistar rats, 2 months old and weighing an average of approximately $166 \mathrm{~g}$ at the beginning of the experiment. The animals were individually housed in wire-mesh steel cages and had ad-lib access to Purina Rodent Laboratory Chow (5001) and deionized water unless otherwise specified. The rats were maintained in a room with an ambient temperature of approximately $23^{\circ} \mathrm{C}$ on a $12: 12$-h light:dark cycle (0700 on).

\section{Apparatus and Procedure}

All rats were deprived of water for $23 \mathrm{~h}$ (at 1200) and continued on ad-lib access to food throughout the experiment. The rats were randomly assigned to receive $30 \mathrm{~min}$ of access (at 1100$)$ to water $(N=10)$, a solution of $0.5-\mathrm{mg} / \mathrm{ml}$ caffeine (anhydrous, Sigma; $N=10$ ), or $5 \% \mathrm{w} / \mathrm{v}$ ethanol (from U.S.P. 95\%; $N=10$ ), each followed by $30 \mathrm{~min}$ of access to water. The fluids were presented in calibrated centrifuge tubes fitted with rubber stoppers and valveless stainless steel spouts. This procedure was repeated for 7 days, with a $1-\mathrm{ml} / \mathrm{kg}$ i.p. injection of saline given immediately prior to fluid access on the last 4 days of this adaptation period. On the next 5 test days, the rats were randomly assigned to receive i.p. injections of $0,0.5,1.0,2.0$, or $4.0 \mu \mathrm{g} / \mathrm{kg}$ of CCK-8 (gift of Squibb Institute for Medical Research, SQ 19,844, Lot NN025NC), until each rat had received all doses. During the initial 30-min fluid access, the behaviors of each rat were observed and classified once each minute during a tone-cued 0.6 -sec interval, as described by Antin et al. (1975). The categories of behavior that were observed and quantified included feeding, drinking, grooming, sniffing, locomotion, rearing, standing, and resting. To simplify analyses, counts of resting and standing were later combined into an "inactive" category, and counts of grooming, sniffing, rearing, and locomotion were combined into an "active", category. The data were analyzed with split-plot analyses of variance, followed by Duncan's test, at an alpha significance level of $p<.05$.

\section{RESULTS}

Fluid intake is shown in Figure 1, as a function of i.p. dose of CCK-8 for groups of rats with access to caffeine, ethanol, or water. Analysis revealed statistically significant effects of type of fluid $[F(2,27)=16.43, p<.05]$ and dose of CCK-8 $[F(4,108)=3.43, p<.05]$. Duncan's post hoc tests indicated that the rats that received water consumed significantly $(p<.05)$ more fluid than did the rats with access to caffeine or ethanol; the latter groups 


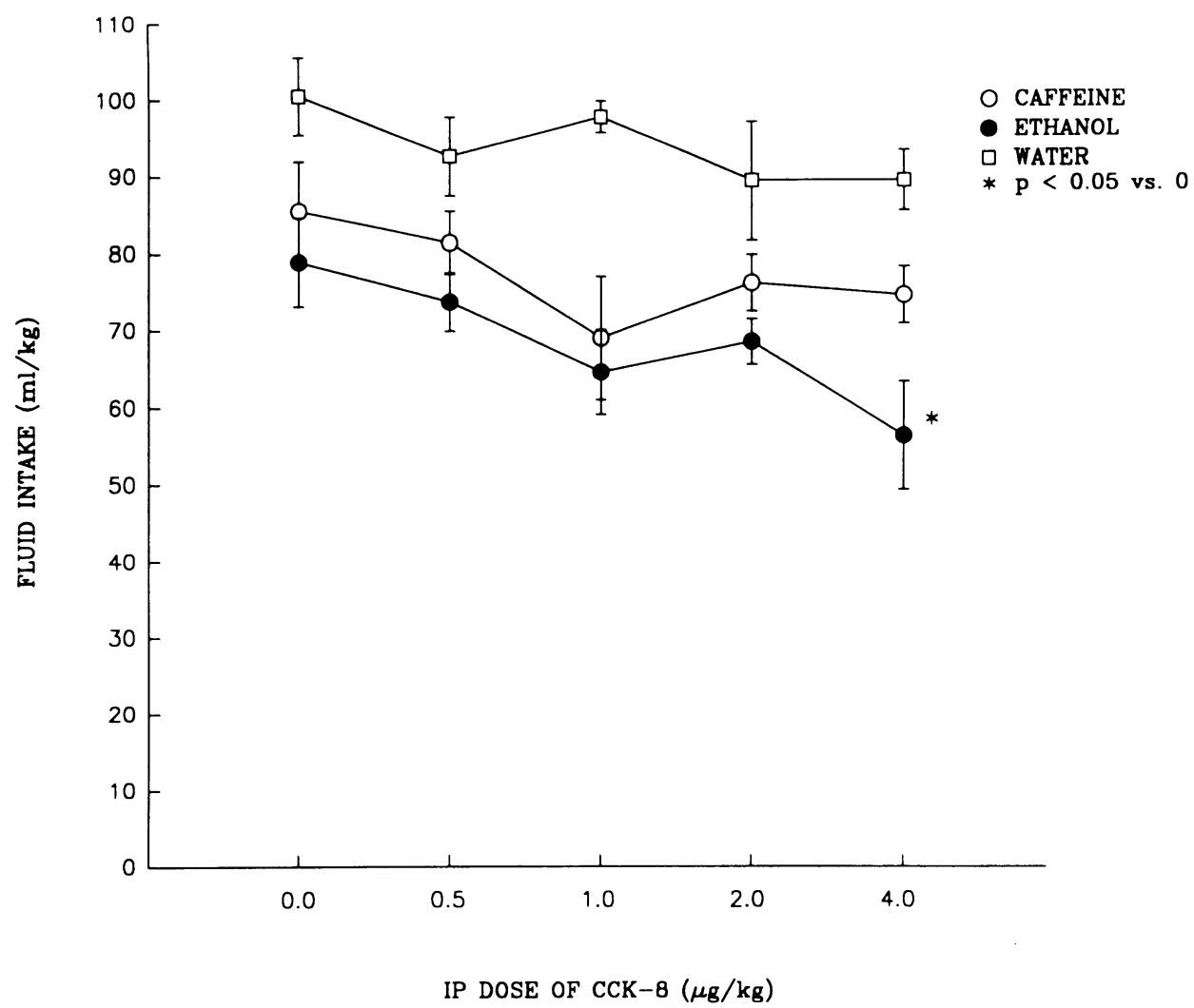

Figure 1. Mean ( $\pm S E$ ) fluid intake (in milliliters/kilogram) of water-deprived rats $(N s=10)$ receiving caffeine $(0.5 \mathrm{mg} / \mathrm{ml}), 5 \% \mathrm{w} / \mathrm{v}$ ethanol, or water, as a function of i.p. dose of cholecystokinin octapeptide (in micrograms/kilogram), during a 30-min session.

did not differ reliably $(p>.05)$. CCK-8 significantly reduced mean intake of $5 \%$ ethanol only, by $-28.6 \%$ at a dose of $4.0 \mu \mathrm{g} / \mathrm{kg}(p<.05)$. Analysis of the separate categories of observed behavior revealed no significant main effects of groups or of CCK-8 dose for the categories of drinking, grooming, locomotion, rearing, standing, and resting, nor were there any significant interactions between them (all $p s>.05$ ). However, there was a significant effect of group on feeding behavior $[F(2,27)=7.15$, $p<.05]$.

Mean percentage of total observations made in the category of feeding is displayed in Figure 2 as a function of i.p. dose of CCK-8, for groups of rats receiving caffeine, ethanol, or water. Mean feeding behavior of the group receiving caffeine was significantly lower than feeding of the groups that received ethanol or water $(p<.05)$; the latter groups did not differ reliably. A Duncan's test showed a tendency of CCK-8 to reduce observed feeding at $4.0 \mu \mathrm{g} / \mathrm{kg}$ in the ethanol group $(p=.05)$. Reanalysis of data into 5-min time blocks showed that CCK-8 reliably suppressed feeding in this group at each dose 11-15 min after injection.

When observations of resting and standing were combined into an "inactive"' category, there were no signifi- cant effects of group and CCK-8 dose ( $p$ s $>.05$ ), nor was there a significant interaction. However, when observations of grooming, sniffing, rearing, and locomotion were combined into an "active" category, there was a significant effect of group $[F(2,27)=7.60, p<.05]$. As can be seen in Figure 3, the rats that received caffeine displayed significantly more "active" behaviors than did the rats that received ethanol or water $(p<.05)$.

\section{DISCUSSION}

The results of this experiment indicate that i.p. injection of CCK-8 decreases intake of ethanol solution, but not intake of water or caffeine solution. That CCK-8 administration reduces intake of alcohol but not water has been demonstrated previously in between-subjects one-bottle tests (Kulkosky, 1984) and within-subjects two-bottle tests (Kulkosky, Foderaro, Sandoval, Cesar, \& Marrinan, 1991; Kulkosky, Sanchez, Foderaro, \& Chiu, 1989; Toth, Shaw, Perlanski, \& Grupp, 1990). The present results further indicate that CCK-8 does not reduce the consumption of a caffeine solution that is psychoactive but noncaloric. Thus CCK-8's action appears to be specific to caloric solutions and is not attributable to a general hypodipsia, debilitation, conditioned aversion, or general drug satiation effect.

A prominent finding in the present study is that orally consumed caffeine strongly suppresses feeding behavior in the rat. Injected caffeine has been shown previously to inhibit food intake at high doses (Dietze \& Kulkosky, 1991; McKim, 1980). Intake of caffeine by water-deprived 


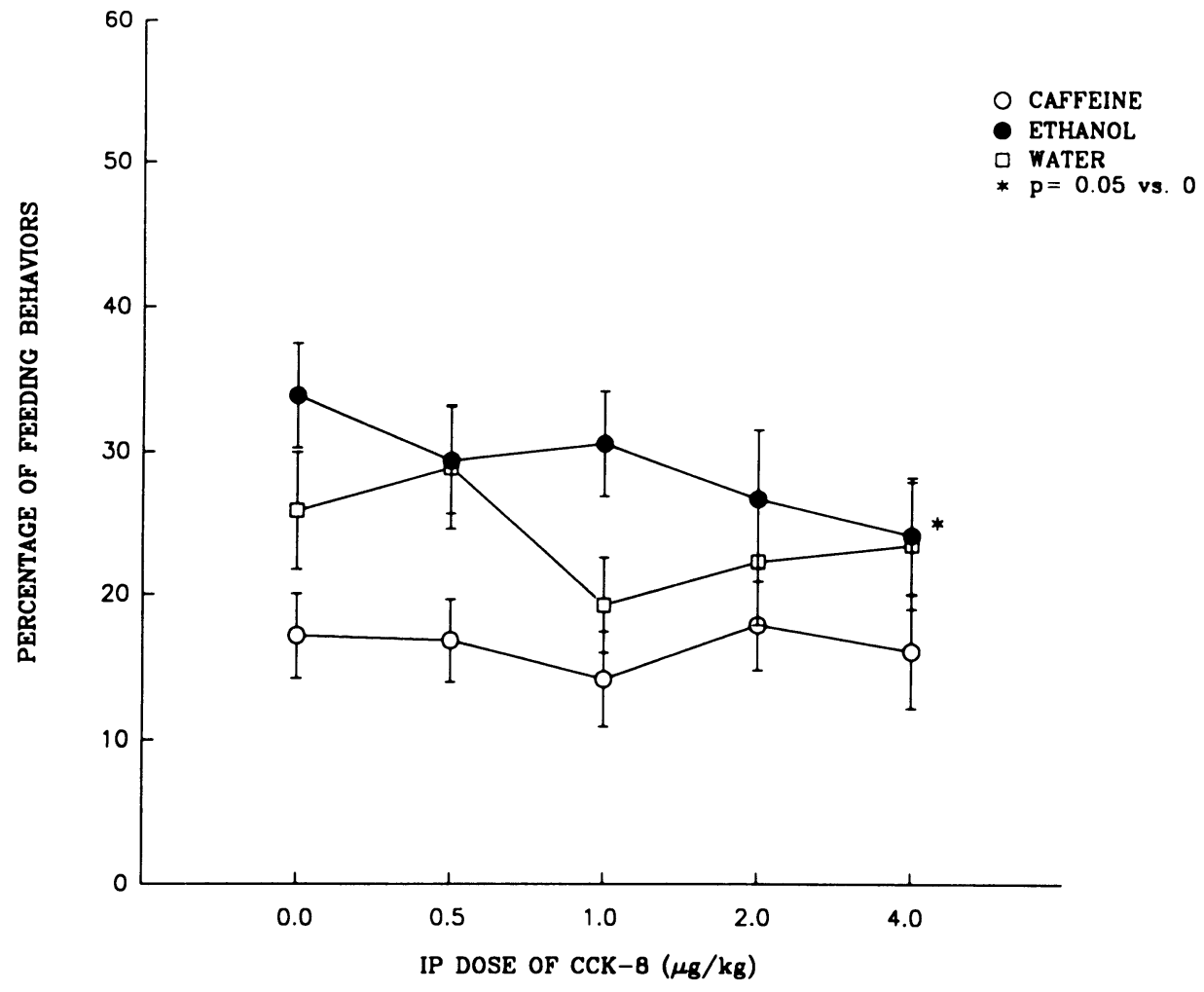

Figure 2. Mean $( \pm S E$ ) percentage of total observations made in the category of feeding behavior of rats receiving caffeine, ethanol, or water, as a function of $i$.p. dose of cholecystokinin octapeptide, during a 30-min session.

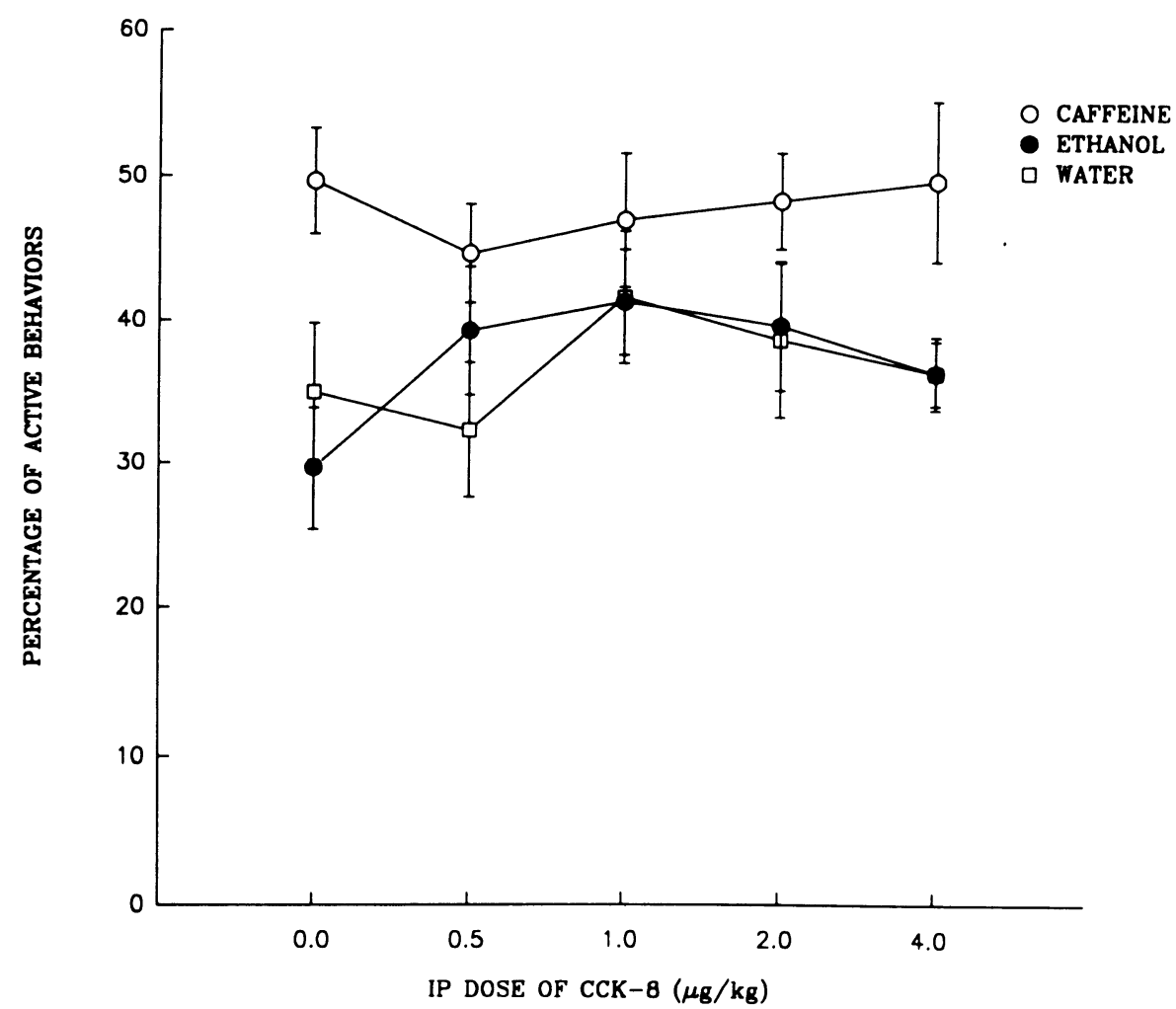

Figure 3. Mean $( \pm S E$ ) percentage of total observations made in the category of active behaviors (grooming, snifing, locomotion, and rearing) of rats receiving caffeine, ethanol, or water, as a function of i.p. dose of cholecystokinin octapeptide, during a 30-min session. 
rats averaged $39 \mathrm{mg} / \mathrm{kg}$ in the 30 -min session, which is within the range of doses that inhibit food intake after caffeine injection. However, CCK-8 injection did not influence the feeding of rats consuming caffeine, which suggests either a blocking of CCK-8's effect by caffeine or a floor effect produced by caffeine-induced anorexia. A floor effect or insensitivity of the dependent variable of observed feeding to CCK-8's action seems more likely in view of the lack of a reliable decrease in feeding after CCK-8 in the group that received water. A similar insensitivity of the observed behavior can be seen in the lack of decrease in observed drinking after CCK-8 in the ethanol group, although intake reliably decreased. This lack of correlation of observed drinking and ethanol intake after CCK-8 injection has been reported earlier (Kulkosky, Sanchez, \& Glazner, 1986), and it has been explained by the decreased licking efficiency reported after CCK-8 injection (Hsiao \& Spencer, 1983). Caffeine consumption was also associated with an increase in high activity behaviors, which gives evidence of an effective stimulant action of the oral caffeine.

To conclude, CCK-8 specifically reduces intake of ethanol solution in accord with a hypothesized caloric satiation action. Oral caffeine robustly suppresses feeding, but this anorexia is not influenced by CCK-8. The chemically induced anorexias of CCK- 8 and caffeine appear to be nonadditive and behaviorally distinct.

\section{REFERENCES}

Antin, J., Gibbs, J., Holt, J., Young, R. C., \& Smith, G. P. (1975). Cholecystokinin elicits the complete behavioral sequence of satiety in rats. Journal of Comparative \& Physiological Psychology, 89, 784-790.

Dietze, M. A., \& Kulkosky, P. J. (1991). Effects of caffeine and bombesin on ethanol and food intake. Life Sciences, 48, 1837-1844.

GibBS, J., \& SMrTH, G. P. (1991). Cholecystokinin and satiety: Problems in brain-gut interactions. In Y. Taché \& D. Wingate (Eds.), Braingut interactions (pp. 255-262). Boca Raton, FL: CRC Press.

GibBs, J., Young, R. C., \& Smith, G. P. (1973). Cholecystokinin decreases food intake in rats. Journal of Comparative \& Physiological Psychology, 84, 488-495.

Hederra, A., Aldunate, J., Segovia-Riquelme, N., \& Mardones, J.
(1975). Effect of caffeine on the voluntary alcohol intake of rats. In J. D. Sinclair \& K. Kiianmaa (Eds.), The effects of centrally active drugs on voluntary alcohol consumption (pp. 9-13). Helsinki: Finnish Foundation for Alcohol Studies.

Hsino, S., \& SPENCER, R. (1983). Analysis of licking responses in rats. Effects of cholecystokinin and bombesin. Behavioral Neuroscience, 97, 234-245.

KULKOSKY, P. J. (1984). Effect of cholecystokinin octapeptide on ethanol intake in the rat. Alcohol, 1, 125-128.

KuLKosKY, P. J. (1985). Brain-gut neuropeptides and the limitation of ethanol consumption. Neuroscience \& Biobehavioral Reviews, 9, 179-190.

Kulkosky, P. J., Foderaro, M. A., Sandoval, S. L., Cesar, S. S., a Marrinan, D. A. (1991). Cholecystokinin-induced satiation with ethanol: Effects of lighting cycle and limited access procedures. Alcohol, 8, 223-227.

Kulkosky, P. J., Sanchez, M. R., Foderaro, M. A., \& Chiu, N. (1989). Cholecystokinin and satiation with alcohol. Alcohol, 6, 395-402.

Kulkosky, P. J., SAnchez, M. R., \& Glazner, G. W. (1986). Cholecystokinin octapeptide: Effect on the ethogram of ethanol consumption and blood ethanol levels in the rat. Physiological Psychology, 14, 23-30.

MCKIM, W. A. (1980). The effect of caffeine, theophylline and amphetamine on operant responding of the mouse. Psychopharmacology, 68, 135-138.

Mueller, K., \& Hsino, S. (1977). Specificity of cholecystokinin satiety effect: Reduction of food but not water intake. Pharmacology, Biochemistry \& Behavior, 6, 643-646.

SMITH, G. P. (1984). Gut hormone hypothesis of postprandial satiety. In J. A. Stunkard \& E. Stellar (Eds.), Eating and its disorders (pp. 67-75). New York: Raven Press.

SmITH, G. P., \& GiBBS, J. (1988). The satiating effect of cholecystokinin. In M. Winick (Ed.), Control of appetite (pp. 35-40). New York: Wiley.

Toth, P., Shaw, C., Perlanski, E., \& Gupp, L. A. (1990). Cholecystokinin octapeptide reduces ethanol intake in food- and watersated rats. Pharmacology, Biochemistry \& Behavior, 35, 493-495.

(Manuscript received April 18, 1991.) 